\title{
Variante morfológica de adultos hembras de Anopheles benarrochi (Diptera: Culicidae) en Putumayo, Colombia
}

\author{
Martha L. Quiñones ${ }^{1}$, Ralph E. Harbach ${ }^{2}$, David A. Calle ${ }^{1}$, Fredy Ruiz ${ }^{1}$, \\ Holmes F. Erazo ${ }^{3}$, Yvonne-Marie Linton ${ }^{2}$ \\ ${ }^{1}$ Programa de Estudio y Control de Enfermedades Tropicales, PECET, Facultad de Medicina, \\ Universidad de Antioquia, Medellín, Colombia. \\ 2 The Mosquitoes Programme and Biomedical Sciences Theme, Department of Entomology, The \\ Natural History Museum, London, United Kingdom. \\ ${ }^{3}$ División Administrativa de Salud, DASALUD, Putumayo, Colombia.
}

Con el propósito de ampliar el conocimiento sobre las especies de Anopheles presentes en el Putumayo, sur de Colombia, y para esclarecer la identidad de los ejemplares clasificados como Anopheles (Nyssorhynchus) evansae en esta región, se recolectaron mosquitos hembra en cebo humano, se alimentaron en pequeños mamíferos y se mantuvieron vivos para la cría de isofamilias. Se realizaron observaciones de las características morfológicas de los huevos, larvas, pupas y adultos de ambos sexos, incluidas las genitalias masculinas. Se obtuvieron 247 posturas de madres identificadas preliminarmente como A. (N.) evansae. A 27 de estas familias se les estudió la morfología de los estadios asociados. Todos los especímenes fueron subsecuentemente identificados como Anopheles (N.) benarrochi por la morfología de los huevos, larvas, pupas y genitalias masculinas, lo cual coincidió con las descripciones publicadas para esta especie. Sin embargo, los adultos hembra de Putumayo presentaron la proporción oscura en el tarsómero posterior 2, entre 0,17 y 0,33 de su longitud, inferior a lo informado para esta especie, lo cual se superpone con los rangos de Anopheles (N.) oswaldoi y $A$. (N.) evansae. Como resultado de la superposición en este carácter, es probable que hembras adultas de $A$. (N.) benarrochi hayan sido incorrectamente identificadas como $A$. (N.) evansae y $A$. (N.) oswaldoi en el sur de Colombia. La presencia de esta variante morfológica ha dificultado la identificación de $A$. (N.) benarrochi en Putumayo y, probablemente, en otras regiones de Colombia y países vecinos.

Palabras clave: Anopheles benarrochi, Nyssorhynchus, taxonomía, Putumayo.

Morphological variation in Anopheles benarrochi (Diptera: Culicidae) adult females specimens from Putumayo, Colombia

A survey of Anopheles mosquitoes was undertaken in Putumayo, southern Colombia, to clarify the identity of specimens classified as Anopheles (N.) evansae in the region. Female anophelines were captured on human bait; then blood fed on small mammals to obtain eggs for progeny broods. Morphological characteristics of the eggs, larvae, pupae and adults of both sexes, including male genitalia were examined. Eggs were obtained from 247 females provisionally identified as $A$. (N.) evansae. Morphology of associated life stages of the progeny broods was compared among 27 families. The morphology of all stages corresponded to the published descriptions of Anopheles (N.) benarrochi. However, morphologically variable adult females were noted. In hind tarsomere 2, the dark portion ranged from 0.17 to 0.33 of the tarsomere length; this is less than previously reported for $A$. (N.) benarrochi and overlaps the ranges described for $A$. (N.) oswaldoi and $A$. (N.) evansae. This variable character probably has led to incorrect identifications of adult $A$. (N.) benarrochi females as $A$. (N.) evansae or $A$. (N.) oswaldoi in specimens from southern Colombia. As a consequence, A. (N.) benarrochi has not been previously recognized in Putumayo, and probably has been misidentified in other areas of northwestern South America.

Key words: Anopheles benarrochi, Nyssorhynchus, taxonomy, Putumayo. 
Las especies del subgénero Nyssorhynchus del género Anopheles se encuentran frecuentemente en simpatría y muestran un alto grado de similaridad morfológica interespecífica en sus estadios adultos y unos niveles considerables de variabilidad intraespecífica que hace difícil su determinación taxonómica. Actualmente, las claves más usadas para la determinación de especies de este subgénero son las de Faran (1) y Faran y Linthicum (2), quienes realizaron una revisión completa de las secciones Albimanus y Argyritarsis de Nyssorhynchus. En Colombia se hizo una traducción y adaptación de la clave de Faran y se incluyeron especies de otros subgéneros en una clave (3), la cual se usa frecuentemente en las seccionales de salud para la determinación de las especies de Anopheles. De acuerdo con Faran (1), las características más confiables para la determinación de especies del subgénero Nyssorhynchus son la morfología de las genitalias masculinas y la quetotaxia de las larvas de cuarto estadio. En estudios epidemiológicos, el interés fundamental es conocer las especies antropofílicas que, por consiguiente, puedan estar involucradas en la transmisión de malaria. También es de importancia fundamental identificar las especies, principalmente los vectores, en evaluaciones de medidas de control y en vigilancia de la resistencia a insecticidas (4). Los muestreos de los estadios inmaduros (larvas o pupas) o de machos, no proveen información sobre el grado de asociación entre estas especies y los humanos. Para obtener esta información, es esencial recolectar e identificar hembras adultas capturadas en el momento de picar al hombre o en reposo en el domicilio. Es justo en este estadio que la correcta identificación de algunas especies del subgénero Nyssorhynchus, con el uso de las claves existentes $(1,2)$, es extremadamente difícil y problemática.

En el departamento del Putumayo, en el sur de Colombia, se ha informado la presencia de varias especies de Anopheles. Estas incluyen, en orden

\footnotetext{
Correspondencia:

Martha Quiñones

martqp@yahoo.com

English translation of the paper available on request.

Recibido: 07/09/01; aceptado: 23/11/01
}

alfabético por subgénero: A. (Anopheles) apicimacula Dyar y Knab, A. (A.) costai Da Fonseca y Da Silva Ramos (como $A$. mediopunctatus) (5), A. (A.) fluminensis Root, $A$. (A.) mattogrossensis Lutz y Neiva, A. (A.) neomaculipalpus Curry, A. (A.) punctimacula Dyar y Knab, A. (A.) peryassui Dyar y Knab, A. (A.) squamifemur Antunes, A. (Nyssorhynchus) argyritarsis Robineau-Desvoidy, $A$. (N.) braziliensis (Chagas), A. (N.) darlingi Root (sólo en los límites con Amazonas), A. (N.) evansae (Brethes), A. (N.) marajoara Galvao y Damasceno (como $A$. albitarsis) (6), A. (N.) oswaldoi (Peryassu), A. (N.) rangeli Gabaldón, Cova Garcia y Lopez, $A$. (N.) strodei Root y A. (N.) triannulatus (Neiva y Pinto) (7, Registros de la Sectretaría de Salud de Putumayo, I. Palacios, comunicación personal).

En el sur de Colombia, las especies $A$. (N.) evansae y $A$. (N.) rangeli son las que con mayor frecuencia pican al hombre $(7,8)$. La determinación de A. (N.) evansae se ha realizado con base en las características morfológicas de hembras adultas y en las claves existentes (1-3). Sin embargo, la distribución informada para esta especie en el estudio más extenso sobre distribución de Nyssorhynchus en Latinoamérica, Faran (1) y Faran y Linthicum (2) informan sobre la presencia de $A$. (N.) evansae en Brasil, Bolivia y Argentina pero no en Colombia. Es frecuente encontrar registros de otras áreas en Colombia en donde la especie A. (N.) evansae hace parte de la entomofauna de anofelinos (9), incluidos Putumayo (10), Casanare (11), Amazonas (12), Guainía (13) y la región de la Costa Pacífica (14). Estos registros sobre la presencia de $A$. (N.) evansae en el país suscitan dudas sobre la correcta identificación de esta especie y la solidez de los estudios de caracteres diferenciales entre especies sobre los cuales están basadas las claves existentes para adultos hembras de Nyssorhynchus. Por consiguiente, la identidad de los especímenes identificados como A. (N.) evansae, tanto en Putumayo como en otros departamentos de Colombia, es incierta. Este estudio se realizó con el propósito de ampliar el conocimiento sobre las especies de Anopheles presentes en el Putumayo y, fundamentalmente, de esclarecer la identidad de los ejemplares identificados como $A$. (N.) evansae en esta región. 


\section{Materiales y métodos}

Se realizaron recolecciones de mosquitos adultos, atraídos a cebo humano en las noches, de las 18:00 a las $21: 00$ horas, dentro y fuera de las viviendas. Las recolecciones se realizaron en diferentes localidades del municipio de Puerto Asís $\left(0^{\circ} 32^{\prime \prime} \mathrm{N} ; 76^{\circ} 31^{\prime \prime} \mathrm{O}\right)$ en el departamento del Putumayo. Los ejemplares recolectados se alimentaron con sangre de hámster, conejo o ratón y se transportaron vivos al Laboratorio de Entomología del PECET, Universidad de Antioquia. En el laboratorio, se criaron las iso-familias a partir de las posturas de cada hembra, hasta obtener los estadios de adulto de los hijos, siguiendo la metodología de Belkin et al. (15). De cada familia se preservó una muestra de los huevos en glutaraldehído al $2 \%$ para microscopía electrónica. Las larvas de cuarto estadio se individualizaron y se recolectaron las pieles de larva y pupa en alcohol al $70 \%$. Las pieles se montaron y se marcaron con un número que permitió asociarlas con su respectivo adulto. Los adultos se montaron en alfiler y a los machos se les realizó montaje de sus genitalias $(1,16)$.

A una muestra de huevos de 5 familias se les tomó fotografías en microscopio electrónico (17) y en el microscopio de luz se observaron muestras de huevos de todas las familias. Los huevos para microscopía electrónica se deshidrataron en gradientes de etanol del 30 al $100 \%$, secado a punto crítico con $\mathrm{CO}_{2}$ y se cubrieron con oropaladio en un cobertor iónico. Finalmente, se observaron en el microscopio electrónico de barrido Hitachi S-510 del Instituto de Biología de la Universidad de Antioquia.

La identificación de las familias se basó en la morfología de las larvas, pupas y adultos, incluidas las genitalias masculinas, utilizando las descripciones y las claves de Faran (1) y Faran y Linthicum (2). La morfología de huevos se comparó con las descripciones publicadas (18-20) para corroborar las identificaciones.

\section{Resultados}

Se obtuvieron huevos de 247 hembras, las cuales fueron identificadas preliminarmente como $A$. (N.) evansae usando la clave de Faran (1). La progenie de 98 familias llegó hasta el estadio adulto y 27 de estas familias se revisaron en el presente estudio. Con la excepción de los adultos hembra, la morfología de las larvas, pupas y genitalias masculinas coincidieron con las descripciones de la especie $A$. (N.) benarrrochi de Faran (1). Las observaciones de la morfología de los huevos también indicaron que las posturas correspondian en su totalidad a $A$. ( $N$.) benarrochi, basadas en las descripciones de Lounibos et al. (20). A continuación se presentan las principales características de esta especie en cada estadio.

Huevo: los huevos de $A$. (N.) benarrochi han sido descritos en detalle por Lounibos et al. (20). Los huevos de esta especie se diferencian de los de otras especies del subgénero Nyssorhynchus por la presencia de una corona pequeña, con paredes cortas y en posición ventral en la parte anterior (figura 1A). Estas caracteristicas fueron evidentes aun bajo el microscopio de luz.

Larva: la morfología de las larvas coincidió completamente con las descripciones de Faran de $A$. (N.) benarrochi (1). En las larvas, las setas 2-C (clipeales internos) (figura 1B), según Faran, pueden ser simples o ramificadas y los 3-C plumosos con hojillas moderadamente largas. En todos los ejemplares revisados en este estudio (53 pieles), las setas 2 y $3-C$ fueron siempre ramificadas, similares a las de $A$. (N.) oswaldoi. Dada esta similaridad es probable que larvas de $A$. (N.) benarrochi y A. (N.) oswaldoi se puedan confundir. Sin embargo, una característica que diferencia a estas dos especies es el tamaño de la seta 1-A (pelo antenal), la cual en $A$. (N.) benarrochi es larga, cerca de dos veces el ancho de la antena en su punto de inserción, mientras que en $A$. (N.) oswaldoi esta seta es corta, sólo una vez el ancho de la antena en su punto de inserción (figura 1B).

Pupa: la morfología de las pupas coincidió con las descripciones de $A$. (N.) benarrochi de Faran (1). La pupa de $A$. (N.) benarrochi presenta la seta 9 del segmento abdominal VII usualmente menor o igual a 0,33 de la longitud del segmento. Esta característica separa a $A$. (N.) benarrochi, $A$. (N.) oswaldoi y $A$. (N.) nuneztovari del resto de 


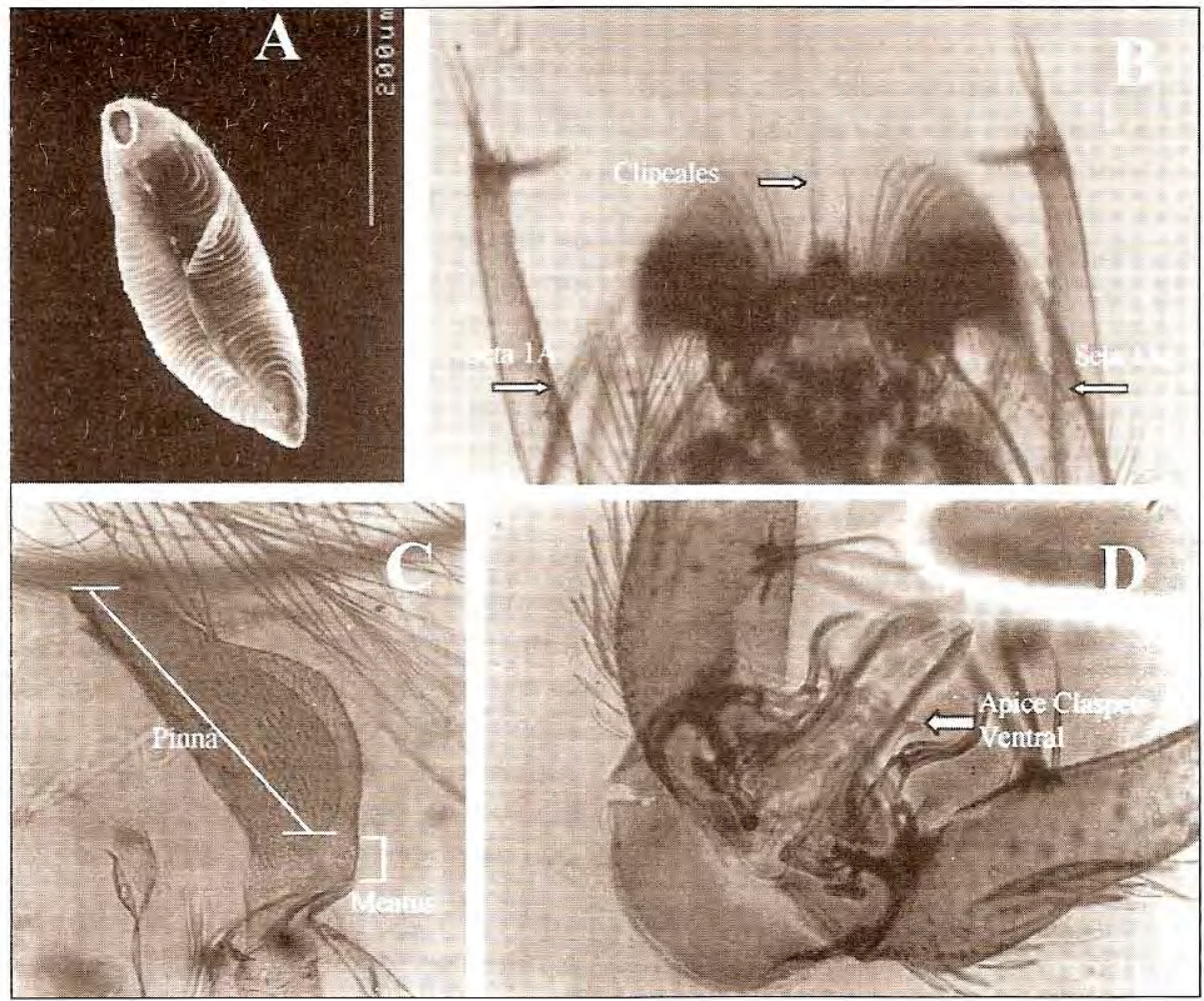

Figura 1. Detalles en los estadios inmaduros y la genitalia del macho de A. (N.) benarrochi. A: Apariencia de los huevos. B: Detalles de la larva en donde se muestran las ramificaciones de las setas 2 y 3-C y la seta 1-A. C: Detalles de la trompeta de las pupas que muestra la apariencia de la pinna con relación al meatus. D: Detalles de la genitalia masculina, particularmente el ápice de la claspeta ventral.

especies de la sección Albimanus. La pupa de $A$. (N.) benarrochise diferencia de $A$. (N.) oswaldoiy $A$. (N.) nuneztovariprincipalmente por la relación entre la longitud del meatus y la longitud de la pinna (figura 1C). Esta relación es de 4,5 a 5,5 en A. (N.) benarrochi, de 1,6 a 2,1 en A. (N.) oswaldoi y de 3,5 a 4,5 en $A$. (N.) nuneztovari.

Genitalia masculina: la morfología de las genitalias masculinas coincidió con las descripciones de $A$. (N.) benarrochi de Faran (1). La genitalia masculina de $A$. (N.) benarrochi se diferencia de las otras especies de la sección Albimanus en que ésta posee una claspeta ventral pequeña, cerca de 0,33 de la longitud del gonocoxito. El ápice de la claspeta es rugosa, expandida en la margen lateral, termina en forma aguda (figura 1D) y posee un lóbulo basal angosto.

Adulto: las observaciones de los adultos hembras revelaron diferencias con las descripciones de $A$.
(N.) benarrochi de Faran (1). En el cuadro 1 se presentan los promedios y rangos encontrados para las principales características usadas en la discriminación de especies de Nyssorhynchus y, particularmente, las usadas en la clave de Faran (1) y Faran y Linthicum (2). La diferencia más importante fue la proporción oscura en el tarsómero posterior 2, la cual cubrió 0,17 a 0,33 de la longitud del tarsómero, proporción muy inferior a la informada por Faran (1) y Faran y Linthicum (2) (cuadro 1). En la figura $2 A$ se muestra la apariencia del tarsómero posterior 2 en $A$. (N.) benarrochi del Putumayo. Otras características estudiadas en el estadio adulto fueron, en el ala, principalmente, el tamaño de la mancha humeral clara con relación a la longitud de la basal oscura, la cual presentó una mayor variabilidad comparada con los registros de Faran (1), lo mismo que el tamaño de la subcostal clara con relación a la subcostal oscura (cuadro 1, figura 2B). 


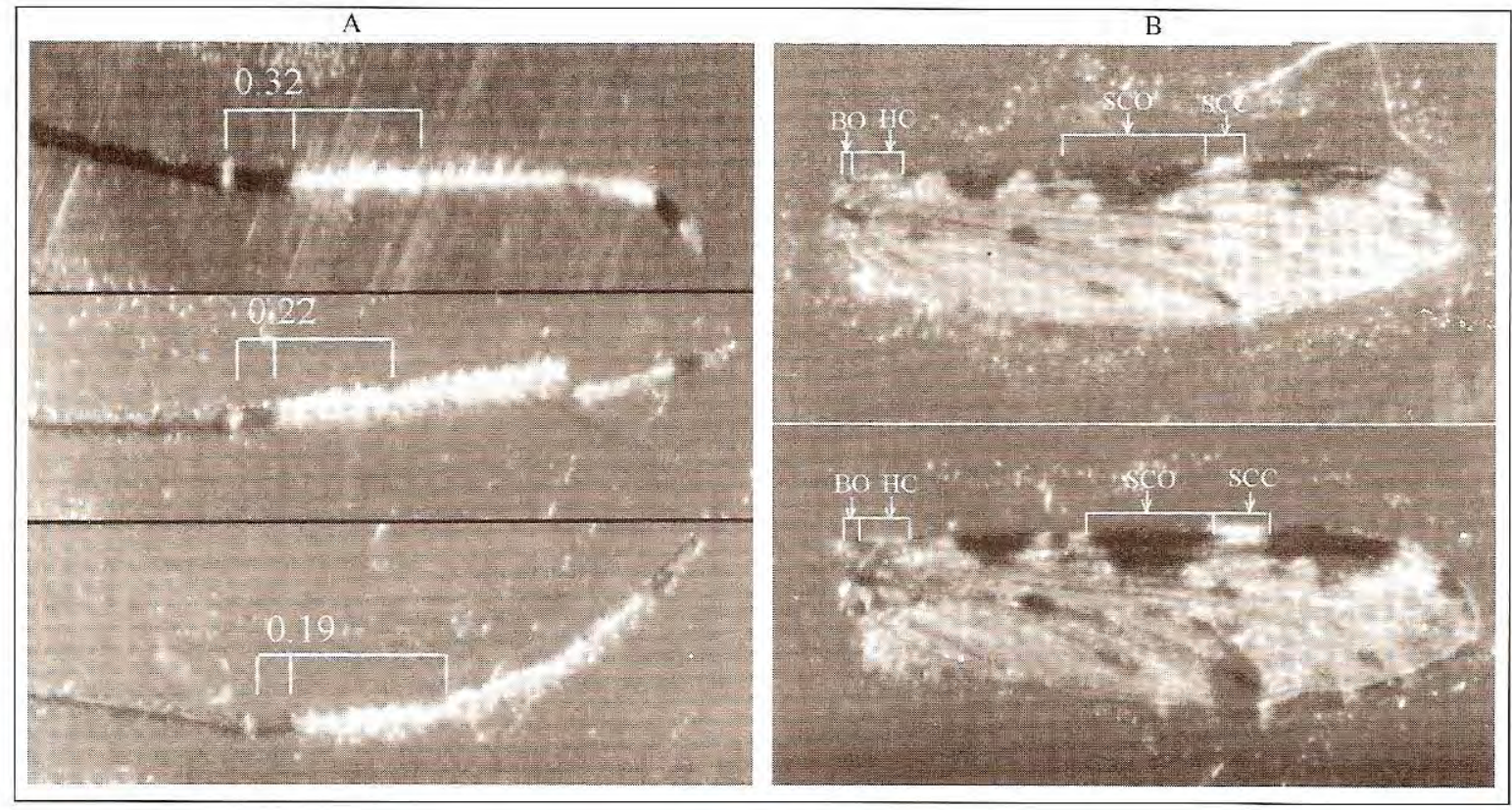

Figura 2. Detalles de las características de las patas posteriores y alas en los adultos hembras de $A$. (N.) benarrochi de Putumayo. A: Tarsómero posterior 2, que muestra la proporción oscura con relación al tamaño del tarsómero. B: Alas con las manchas de la costa, en las que se muestra, particularmente, la apariencia de la mancha basal oscura (BO), humeral clara $(\mathrm{HC})$, subcostal oscura (SCO) y la subcostal clara (SCC).

\section{Discusión}

En Colombia, la especie $A$. (N.) benarrochi fue registrada por el SEM (21), entre 1944 y 1957, en Caldas, Cundinamarca, Santander, Meta y Putumayo. En los Llanos Orientales, fue registrada por Renjifo y De Zuleta (22). Faran (1) registra esta especie en Colombia y Venezuela, principalmente en la región de la Orinoquia. Se registra también en el occidente de Venezuela (23), en Loreto, Perú (1), al norte de Brasil en la región amazónica y en el sur de Brasil en el estado de Sao Paulo (24). En el departamento del Putumayo, en registros posteriores a 1957, la especie $A$. (N.) benarrochi ya no hace parte de la entomofauna de anofelinos (7), probablemente, por dificultades en su determinacion. En la revisión de Faran (1), con material procedente de Villavicencio, Colombia, se menciona que la morfología de hembras adultas de $A$. (N.) benarrochi es muy similar a $A$. (N.) aquasalis Curry y a $A$. (N.) strodei. Inclusive en la descripción original (25), Gabaldon et al. discuten la dificultad de diferenciar los adultos de $A$. (N.) benarrochi de las especies $A$. (N.) strodei, $A$. (N.), nuneztovari Gabaldón y $A$. (N.) rangeli.
Con los resultados del presente estudio es claro que $A$. (N.) benarrochi ha sido erróneamente identificado como $A$. (N.) evansae o como A. (N.) oswaldoi en Putumayo, debido a la presencia de esta variante morfológica en su estadio adulto, la cual presenta la mancha oscura del tarsómero posterior 2 inferior a la de las descripciones $(1,2)$. Esta característica de los adultos hembra se usa frecuentemente en las claves para determinar especies del subgénero Nyssorhynchus. La proporción oscura en el tarsómero posterior 2 en A. (N.) benarrochi de Putumayo se encontró con un rango de entre 0,17 y 0,33 de la longitud del tarsómero. En contraste, las descripciones de Faran (1) y Faran y Linthicun (2) para esta especie indican que este tarsómero tiene aproximadamente la mitad oscura ( 0,40 - 0,55 de longitud), con un rango entre 0,36 y 0,55 . La diferencia en esta característica entre los especímenes del Putumayo y las descripciones y claves (1-3) es importante para entender porqué se venía identificando erróneamente a $A$. (N.) benarrochi. En la clave de Faran (1), la especie $A$. (N.) oswaldoise diferencia de $A$. (N.) rangeli, $A$. (N.) 
Cuadro 1. Medias y rangos de tres características de A. (N.) benarrochi observadas en este estudio, comparadas con los rangos descritos por Faran (1) y sobre los cuales se basa la clave para la identificación de adultos hembras de Nyssorhynchus.

\begin{tabular}{lcccc}
\hline Característica & $\mathbf{n}$ & Promedio & Rango & Informado por Faran \\
\hline $\begin{array}{l}\text { Proporción oscura en el tarsómero } \\
\text { posterior 2 }\end{array}$ & 33 & 0,26 & $0,17-0,33$ & $0,40-0,55(0,36-0,55)$ \\
$\begin{array}{l}\text { Humeral clara con relación a la lorgitud } \\
\text { de la basal oscura }\end{array}$ & 33 & 2,27 & $1,70-3,30$ & $\geq 2,50$ \\
$\begin{array}{l}\text { Subcostal clara con relación la } \\
\text { subcostai oscura }\end{array}$ & 33 & 0,32 & $0,10-0,55$ & $0,40(0,30-0,50)$ \\
\hline
\end{tabular}

benarrochi, A. (N.) trinkae, A. (N.) nuneztovari, A. (N.) evansae y A. (N.) strodei por tener la proporción oscura en el tarsómero posterior 2 inferior a 0,25 de la longitud del tarsómero. Por consiguiente, es probable que algunos ejemplares adultos de $A$. (N.) benarrochi, particularmente los que presentan esta mancha oscura inferior a 0,25 , se hayan identificado erróneamente como $A$. (N.) oswaldoi y que los que presentan esta mancha entre 0,25 y 0,33 se hayan identificado erróneamente como $A$. (N.) evansae con base en las claves disponibles (1-3). Debido a la presencia de esta variabilidad morfológica en las hembras adultas, es probable que $A$. (N.) benarrochi esté siendo erróneamente identificado en estudios de rutina en Latinoamérica. Es necesario desarrollar una clave morfológica actualizada, teniendo en cuenta toda la variabilidad de esta especie, que permita la diferenciación de adultos hembras de A. (N.) benarrochi de los de A. (N.) oswaldoi y A. (N.) evansae.

En su estadio larval, $A$. (N.) benarrochi puede ser fácilmente confundido con $A$. (N.) oswaldoi debido a la semejanza en la morfología de las setas 2 y 3-C (clipeales). Obvias diferencias entre A. (N.) benarrochi y otras especies del subgénero Nyssorhynchus se encuentran en la pupa y la genitalia masculina, pero la revisión de la morfología de estos estadios demanda tiempo y no es muy usada en estudios de rutina. Es posible, por consiguiente, que $A$. (N.) benarrochi pueda estar presente también en otras regiones de Colombia, particularmente en aquellas áreas en que han informado sobre las especies $A$. (N.) evansae junto con $A$. (N.) oswaldoi. Es necesario verificar su presencia observando todos los estadios asociados bien sea a partir de larvas o a partir de la cría de isofamilias. En este estudio fue particularmente útil la observación de la morfología de los huevos, lo cual se puede realizar utilizando un microscopio de luz. Actualmente, se están desarrollando métodos moleculares en nuestro laboratorio para diferenciar $A$. ( $N$.) benarrochi de otros miembros del subgénero Nyssorhynchus (Linton et al., en preparación); estos métodos pueden usarse para identificar la especie en cualquier estadio y sobre ellos se informará en otro artículo.

En la revisión realizada por Ferro (10) y en informes internos del antiguo Servicio de Erradicación de la Malaria se menciona que la especie $A$. (N.) evansae (como A. (N.) noroestensis) es la responsable de la transmisión de malaria en el Putumayo. Este registro se realizó únicamente por ser la especie que pica con más frecuencia a humanos en esta región, sin tener ninguna evidencia de infectividad natural con Plasmodium. Dada la dificultad de determinar correctamente las especies del subgénero Nyssorhynchus cuando sólo se tienen hembras adultas (1), se podría pensar que los ejemplares determinados como $A$. evansae en Putumayo pudieran ser alguna otra especie de este subgénero con reconocido papel vectorial. Sin embargo, en los tres años de muestreo de este estudio sobre la fauna de mosquitos Anopheles en Putumayo, incluida la cría de isofamilias y crías individuales de larvas, no se ha encontrado evidencia de la presencia de especies con reconocido papel vectorial. Estos resultados generan el interrogante sobre cuáles especies son responsables de la transmisión de malaria en esta región. Actualmente, se adelantan estudios de infectividad natural con ejemplares recolectados con cebo humano para determinar 
cuáles especies están involucradas en la transmisión de malaria en Putumayo.

Muy poco se conoce sobre ia biología e importancia médica de $A$. (N.) benarrochi en Colombia. De acuerdo con la información disponible en la revisión de Faran (1), esta especie no parece tener un papel como vector de la malaria y se informa como una especie que raramente entra a las viviendas y se alimenta principalmente de animales. El alto grado de antropofilia observado para la población de $A$. (N.) benarrochi del sur de Colombia abre la posibilidad de que, en ausencia de vectores tradicionales, esta especie pueda estar involucrada en la transmisión de malaria en Putumayo, situación que es necesario estudiar. La presencia de A. ( N.) benarrochien Putumayo constituye una oportunidad para ampliar el conocimiento sobre su bionomía y ecología. Es necesario realizar estudios en otras áreas del país y países vecinos para verificar la identidad de los especímenes determinados como $A$. (N.) evansae usando las claves existentes, evaluar el grado de variabilidad morfológica y confirmar la distribución actual de A. (N.) benarrochi en Latinoamérica. Seria muy interesante estudiar las posibles causas de esta diferencia morfológica entre especímenes de $A$. (N.) benarrochi de Putumayo y de otras regiones en donde su morfología coincide con las descripciones y claves, para tratar de explicar las razones de estas diferencias, principalmente en la longitud de la mancha oscura del tarsómero posterior 2. Es probable que estén involucradas variables ambientales como la temperatura, lo cual se ha descrito para otras especies de Anopheles (26-29). Sin embargo, es común encontrar variabilidad intraespecífica, particularmente en especies del subgénero Nyssorhynchus, aun en la progenie de la misma hembra (30-32), por lo que se podría pensar que las descripciones $y$ claves existentes no cubrieron toda la variabilidad morfológica de $A$. (N) benarrochi.

Los resultados de este estudio muestran claramente la dificultad en la determinación taxonómica de las especies de Anopheles del subgénero Nyssorhyṇchus cuando se emplean solamente hembras adultas. Para lograr una eficiente vigilancia entomológica, es fundamental tener estudios básicos en cada región para conocer con certeza las especies presentes. Estos estudios deben incluir la cría de isofamilias, series, observación de la morfología de los huevos, larvas, pupas y genitalias masculinas, herramientas esenciales en taxonomía. Sólo con una correcta determinación taxonómica de las especies y con el conocimiento de su bionomía y papel en la transmisión de la malaria, es posible diseñar adecuadas medidas de vigilancia, prevención y control dirigidas a los vectores en Colombia.

\section{Agradecimientos}

Este estudio se realizó con financiación de The Wellcome Trust, DASALUD, Colciencias y CODI de la Universidad de Antioquia. Queremos expresar nuestros agradecimientos a Iván Gerardo Guerrero, director de DASALUD, por el apoyo a este estudio; a William Galarza, Edmundo Caicedo e Ignacio Palacios de DASALUD por su interés, participación y coordinación de las actividades de campo; a los señores del Grupo de Entomología del Putumayo, DASALUD, Remigio Solarte, Carlos Andrés Pérez (qepd), Marcos Pérez (qepd), Eliécer Gualguan y Rafael Burbano por su invaluable ayuda en las recolecciones de campo, sin quienes este estudio no se hubiera podido realizar; a Iván Darío Vélez, director del PECET, Facultad de Medicina, por su constante apoyo; a Dora Amparo Estrada por el montaje de las pieles y fotografías de los huevos en microscopio electrónico y a Edison Correa (qepd) por su entrega a este proyecto, el cual le costó la vida.

\section{Referencias}

1. Faran ME. Mosquito studies (Diptera: Culicidae) XXXIV. A revision of the Albimanus section of the subgenus Nyssorhynchus of Anopheles. Contribution of the American Entomological Institute 1980;15:1-214.

2. Faran ME, Linthicum KJ. A handbook of the Amazonian species of Anopheles (Nyssorhynchus) (Diptera: Culicidae). Mosquito Systematics 1981;13:1-18.

3. Suárez MF, Quiñones ML, Fleming GA, Robayo M. Guía introductoria a la morfología de Anopheles y clave para determinación de las principales especies de Colombia. Bogotá, Colombia: Dirección de Campañas Directas, Ministerio de Salud; 1989.

4. Elliot R. The influence of vector behaviour on malaria transmission. Am J Trop Med Hyg 1972;21:755-63. 
5. Sallum MAM, Wilkerson RC, Forattini OP. Taxonomic study of species formerily identified as Anopheles mediopunctatus and resurrection of $A$. costai (Diptera: Culicidae). J Med Entomol 1999;36:282-300.

6. Linthicum K. A revision of the Argyritarsis section of the subgenus Nyssorhynchus of Anopheles (Diptera: Culicidae). Mosquito Systematics 1998;20:98-271.

7. Suárez MF, Quiñones ML, Wirtz WA. Anopheles rangeli, a suspected vector of Plasmodium vivax in southern Colombia. Abstracts, The $39^{\text {th }}$ Annual Meeting of the American Society of Tropical Medicine and Hygiene, New Orleans, LA; 1990. p.158.

3. Quiñones ML, Linton Y-M, Harbach RE, Estrada DA, Erazo HF, Calle DA, et al. Malaria vector species in southern Colombia: species determination and natural infectivity. Abstracts, volume 1, XVth International Congress for Tropical Medicine and Malaria, Cartagena, Colombia; 2000. p. 108.

9. Herrera S, Suárez MF, Sánchez GI, Quiñones ML, de Herrera M. Uso de la técnica radioinmunoensayo IRMA en Anopheles de Colombia para la detección de esporozoitos de Plasmodium. Colombia Médica 1987;18: 2-6.

10. Ferro CA. Revisión de los recursos aplicables a la lucha contra el paludismo. Revista de la Escuela Nacional de Salud Pública 1979;5:11-8.

11. Quiñones ML, Villarreal LI, Suárez MF. Distribution and biting behaviour of Anopheles species in Casanare, Colombia. Symposium summaries. En. Clark GL, Suárez $M F$, editors. Mosquito vector control and biology in Latin America. A second symposium. American Mosquito Control Association 1992;8:305-6.

12. Pérez L, Suárez M, Murcia L, de la Hoz F, Olano VA, Brochero $\mathrm{H}$, et al. La malaria en el Amazonas: conocimientos, prácticas, prevalencia de parasitemia y evaluación entomológica en mayo de 1997. Biomédica 1999;19:93-102.

13. Villegas ME, Quintero R, Manotas LE. Malaria en el Guainia, 1998: frecuencia y aspectos entomológicos. Revista Colombiana de Entomología 2000;26:43-9.

14. Quiñones ML, Suárez MF, Fleming GA. Distribución y bionomía de los anofelinos de la costa Pacífica de Colombia. Colombia Médica 1987;18:19-24.

15. Belkin JN, Schick RX, Galindo P, Aitken THG. Estudios sobre mosquitos (Diptera: Culicidae) la. Un proyecto para un estudio sistemático de los mosquitos de mesoamérica. Ila. Métodos para coleccionar, criar y preservar mosquitos. Contributions of the American Entomological Institute 1967;1:1-89.

16. Lane J. The preservation and mounting of insects of medical importance. WHO mimeographed document. WHO/VBC/74.502; 1974.

17. Estrada DA. Morfología comparativa de huevos de Anopheles rangeli, Anopheles nuneztovari y Anopheles oswaldoi (Diptera: Culicidae) mediante microscopia electrónica de barrido (tesis). Medellín, Colombia: Universidad de Antioquia; 2000.

18. Linley JR, Lounibos LP. The eggs of Anopheles (Nyssorhynchus) rangeli and Anopheles (Nyssorhynchus) dunhami (Diptera: Culicidae). Mosquito Systematics 1993;25:157-69.

19. Linley JR, Lounibos LP, Conn J, Duzak D, Nishimura N. A description and morphometric comparison of eggs from eight geographic populations of the South American malaria vector Anopheles (Nyssorhynchus) nuneztovari (Diptera: Culicidae). J Am Mosquito Control Assoc 1996;12:275-92.

20. Lounibos LP, Duzak D, Linley JR. Comparative egg morphology of six species of the Albimanus section of Anopheles (Nyssorhynchus) (Diptera: Culicidae). J Med Entomol 1979;34:136-55.

21. Servicio Nacional de Erradicación de la Malaria, SEM. Plan de erradicación de la malaria en Colombia. Bogotá: Ministerio de Salud; 1957.

22. Rengifo S, De Zulueta J. Five years' observations of rurai malaria in eastern Colombia. Am J Trop Med Hyg 1952;1:598-611.

23. Delgado N, Rubio-Palis Y. Identification of Anopheles (Nyssorhynchus) (Diptera: Culicidae) occurring in Western Venezuela. Mosquito Systematics 1993;25:22030.

24. Sallum MAM, Bergo ES, Forattini OP. First record of Anopheles benarrochi Gabaldón, Cova Garcia \& Lopez from the State of Sao Paulo, Southern Brazil. Mem Inst Oswaldo Cruz 1997;92:233-4.

25. Gabaldón A, Cova-Garcia P, Lopez JA. Anopheles (Nyssorhynchus) benarrochi una nueva especie de la subserie Triannulatus. Publicaciones de la División de Malariologia 1941;7:3-24.

26. Hribar L. Effect of larval rearing temperatures on adult female morphology of Anopheles (Nyssorhynchus) albimanus (Diptera:Culicidae). Invertebrate Reproduction and Development 1997;32:85-8.

27. González IA, Cárdenas H, González R, Suárez MF. Efecto de la temperatura sobre la variación fenotípica de la zona costal del ala en adultos de Anopheles (Nyssorhynchus) nuneztovari (Diptera:Culicidae). Resúmenes, XXVI Congreso Sociedad Colombiana de Entomología; 1999.

28. Davis NC. Consideration of variability in the Nyssorhynchus group of the genus Anopheles. Am J Hyg 1962;8: 539-63.

29. Leseur D, Sharp BL, Appleton CC. Dark-scaled areas on adult Anopheles mosquitoes are selectively affected by temperature-related size variation. Med Vet Entomol 1992;6:396-8.

30. Harbach RE, Donaid RR, Manguin S. Variation in the hindtarsai markings of Anopheles darlingi (Diptera: 
Culicidae) in Belize. Mosquito Systematics 1993; 25:192-7

31. Rubio-Palis Y. Caracterización morfométrica de poblaciones del vector de malaria Anopheles (Nyssorhynchus) darlingi Root (Diptera: Culicidae) en
Venezuela. Boletín de Entomología Venezolana 1998;13: 141-72.

32. Hribar L. Costal wing spot variation within and among progeny of single female Anopheles nuneztovari (Diptera: Culicidae). Mosquito Systematics 1995;27:1-15. 